\title{
A hybrid immersed boundary-lattice Boltzmann and finite difference method for bushfire simulation
}

\author{
L. Wang ${ }^{a}{ }_{\oplus}$ and F.-B. Tian ${ }^{a}$ \\ ${ }^{a}$ School of Engineering and Information Technology, University of New South Wales, Canberra ACT, 2600 \\ Email: 1.wang@unsw.edu.au
}

\begin{abstract}
Catastrophic bushfire that destroys the assets and multiple facilities happened many times all over the world in the last two decades. The majority of the most damaging fires involve wind and terrain, in order to predict the spread process of bushfire in various geographical and weather conditions, a hybrid numerical method is proposed for bushfire simulation. The outputs from the numerical simulation of bushfire can be used to guide the extinguishment process and improve the security. The present numerical method includes three parts: fluid solver, heat transfer solver, and immersed boundary method for fluid-structure interaction and heat transfer. Specifically, the multi-relaxation time lattice Boltzmann method is adopted for the dynamics of nonNewtonian flow, with sub-grid viscosity model for large eddy simulation, a geometry-adaptive technique to enhance the computational efficiency and immersed boundary method to achieve no-slip boundary conditions. The heat transfer equation is spatially discretized by a second-order up-wind scheme for the convection term, a central difference scheme for the diffusion term, and a second-order difference scheme for the temporal term. The major contribution of this work is the integration of spatial adaptivity, thermal finite difference method, and fluid flow immersed boundary-lattice Boltzmann method. Several benchmark cases including powerlaw fluid flow and heat transfer around a stationary cylinder and flow around a stationary sphere are used to validate the present method and developed solver. The good agreements achieved by the present method with the published data indicate that the present extension is an efficient way for fluid-structure interaction and heat transfer in fluid flow. In addition, a demonstration considering bushfire-wind-terrain interaction is presented.
\end{abstract}

Keywords: Immersed boundary method, lattice-Boltzmann method, heat transfer, bushfire simulation 
L. Wang and F.-B. Tian, A hybrid immersed boundary-lattice Boltzmann and finite difference method...

\section{INTRODUCTION}

Catastrophic bushfire that destroys the assets and multiple facilities happened many times all over the world in the last two decades. The majority of the most damaging fires involve wind and terrain, in order to predict the spread process of bushfire in various geographical and weather conditions, it is necessary to develop an efficient numerical method.

As an alternative method of computational fluid dynamics (CFD), the lattice-Boltzmann method (LBM) has received remarkable attentions due to its simplicity in parallelized computation (Chen and Doolen, 1998; Tian et al., 2011; Guo and Shu, 2013; Xu et al., 2018). In terms of the numerical simulation of thermal fluid systems, most of the previous thermal LBM models adopt the multi-speed approach (Alexander et al., 1993; Qian. 1993: Chen et al., 1997), in which additional particle speeds are needed to obtain the energy equation at the macroscopic level (Inamuro et al. 2002). The additional particle speeds decrease the computational efficiency and the stability is also limited. In order to keep the simplicity of LBM, Peng et al. proposed a simplified thermal lattice Boltzmann model, neglecting the compression work done by the pressure and viscous heat dissipation (Peng et al., 2003). In this study, an alternative method to solve the heat transfer in fluid flow is presented, which conserves the simplicity and efficiency of LBM. Where, the fluid dynamics and heat transfer are solved independently by LBM and finite difference method, respectively, with multi-relaxation time and geometry-adaptive mesh technique to achieve good stability and computational efficiency, respectively. The dynamic subgrid-scale eddy viscosity model proposed by Lilly (Lilly, 1992) is incorporated into the IB-LBM solver for large-eddy simulation (LES). In addition to the thermal process, the fluid-structure interaction (FSI) is also achieved by using an penalty immersed boundary method, which makes it easy for the present method to handle complex geometries.

\section{NUMERICAL METHODS}

In this paper, the non-Newtonian flow and heat transfer involving complex boundaries are considered, where the LBM is adopted for the fluid solver, the finite difference method is used to solve the heat transfer equation, and the complex no-slip boundaries are achieved by an IB method. The numerical methods are briefly introduced in this section, the details can be found in Ref. (Wang and Tian, 2018).

\subsection{Fluid Solver}

In the MRT-based IB-LBM, the evolution equation of the velocity distribution function $g_{i}$ along the $i$-th direction at position $\boldsymbol{x}$ with BGK approximation is expressed as (Guo and Zheng, 2008)

$$
g_{i}\left(\boldsymbol{x}+\boldsymbol{e}_{i} \Delta t, t+\Delta t\right)=g_{i}(\boldsymbol{x}, t)-\Omega_{i}(\boldsymbol{x}, t)+\Delta t G_{i}, \quad i=0,1, \ldots, 18,
$$

where $\Delta t$ is the time step. The collision operator $\Omega_{i}$ and $G_{i}$, which represent the body force effects on the distribution function, are defined as

$$
\begin{aligned}
\Omega_{i} & =-\left(M^{-1} S M\right)_{i j}\left[g_{j}(\boldsymbol{x}, t)-g_{j}^{e q}(\boldsymbol{x}, t)\right] \\
G_{i} & =\left[M^{-1}(I-S / 2) M\right]_{i j} F_{j}
\end{aligned}
$$

where $M$ is a $19 \times 19$ transform matrix for the D3Q19 model (three dimensional with 19 directions) used here, and $S$ is a non-negative diagonal matrix related to the fluid viscosity. The macro density and momentum are given as follows,

$$
\rho=\sum_{i=0}^{18} g_{i}, \quad \rho \boldsymbol{u}=\sum_{i=0}^{18} g_{i} \boldsymbol{e}_{i}+\frac{1}{2} \boldsymbol{f} \Delta t .
$$

The local equilibrium distribution function $g_{i}^{e q}$ and the force term $G_{i}$ are calculated by

$$
\begin{aligned}
g_{i}^{e q} & =\omega_{i} \rho\left[1+\frac{\boldsymbol{e}_{i} \cdot \boldsymbol{u}}{c_{s}^{2}}+\frac{\boldsymbol{u} \boldsymbol{u}:\left(\boldsymbol{e}_{i} \boldsymbol{e}_{i}-c_{s}^{2} \boldsymbol{I}\right)}{c_{s}^{4}}\right], \\
G_{i} & =\omega_{i}\left[\frac{\boldsymbol{e}_{i}-\boldsymbol{u}}{c_{s}^{2}}+\frac{\boldsymbol{e}_{i} \cdot \boldsymbol{u}}{c_{s}^{4}} \boldsymbol{e}_{i}\right] \cdot \boldsymbol{f}
\end{aligned}
$$


L. Wang and F.-B. Tian, A hybrid immersed boundary-lattice Boltzmann and finite difference method...

where $\omega_{i}$ are the weights (Tian et al., 2011). The sound speed $c_{s}=\Delta x /(\sqrt{3} \Delta t)$, and $f$ is the force acting on the fluid. For two-dimensional simulation, D2Q9 model is adopted. The relaxation time is related to the kinematic viscosity $\nu$ in the Navier-Stokes equations in terms of $\nu=(\tau-0.5) c_{s}^{2} \Delta t$. The rheological equation of state for power-law non-Newtonian fluid considered in this paper is defined by (Tian et al. , 2014) $\nu=m\left(I_{2} / 2\right)^{(n-1) / 2}$, where $m$ is the power-law consistency index, $n$ is the power-law fluid behaviour index, and $I_{2}$ is the second invariant of the rate of strain tensor. Additionally, the multi-block technique developed by Yu et al. ( Yu et al. 2002) is combined with the geometry-adaptive method to enhance the computational efficiency. The dynamic subgrid-scale eddy viscosity model proposed by Lilly (Lilly, 1992) is incorporated into the IB-LBM solver for LES.

\subsection{Heat Transfer Solver}

The governing equation for the heat transfer in the fluid is given as (Abel et al. 2005)

$$
\rho c_{p}\left(\frac{\partial T}{\partial t}+\boldsymbol{u} \cdot \nabla T\right)=\kappa \nabla^{2} T+q
$$

where $T$ is the temperature, $c_{p}$ and $\kappa$ are the specific heat and thermal conductivity coefficient of the fluid, respectively. $q$ is the source term.

The second-order upwind scheme is used to discretize the convection term in Equation ( 7 ) explicitly, expressed as

$$
\begin{array}{ll}
\frac{\partial T}{\partial x}=\frac{1}{2 \Delta x}\left(3 T_{i}-4 T_{i-1}+T_{i-2}\right), & u>0 \\
\frac{\partial T}{\partial x}=\frac{1}{2 \Delta x}\left(-3 T_{i}+4 T_{i+1}-T_{i+2}\right), & u<0 .
\end{array}
$$

A second-order internal central difference scheme is adopted for the spatial discretization on the boundaries. The subscripts indicate the position.

The diffusion term in Equation ( 7 ) is discretized using a second-order explicit scheme, expressed as

$$
\frac{\partial^{2} T}{\partial x^{2}}=\frac{1}{\Delta x^{2}}\left(T_{i-1}-2 T_{i}+T_{i+1}\right)
$$

and the internal difference scheme is also used for the boundary nodes. All the discretizations of $T$ in the $y$ direction is similar to that in the $x$ direction. In addition, a second-order explicit scheme is used for the temporal discretization. Therefore, the heat transfer solver has a second-order accuracy spatially and temporally, which is consistent with the accuracy of the LBM. The heat transfer equation is also solved on the geometry-adaptive Cartesian mesh that used for the fluid dynamics.

\subsection{The IB Method for Fluid-Structure Interaction and Heat Transfer}

The pIB method developed by Kim and Peskin (Kim and Peskin 2007) is used to handle the no-slip boundaries between the rigid body and the fluid. The interaction force between the fluid and the structure can be determined by the feedback law (Kim and Peskin, 2007):

$$
\boldsymbol{F}=\alpha \int_{0}^{t}\left(\boldsymbol{U}_{i b}-\boldsymbol{U}\right) d t+\beta\left(\boldsymbol{U}_{i b}-\boldsymbol{U}\right),
$$

where $\boldsymbol{U}_{i b}$ is the boundary velocity obtained by interpolation at the IB, $\boldsymbol{U}$ is the structure velocity, and $\alpha$ and $\beta$ are large positive free constants. The force acting on the Lagrange structure from the ambient fluid can be taken as a concentrated force acting on the corresponding nodes; thus, it can be added to the body force $f$ in Equation (6). Compared to the sharp-interface method (Tian et al., 2014; Mittal et al., 2008), the pIB method is particularly suitable here, since in this approach all the grid points within the computational domain are treated with a unified equation. The transformation between the Euler and Lagrange variables can be realized by the Dirac delta function. The interpolation of velocity and the spreading of the Lagrange force to the adjacent grid points are expressed as

$$
\begin{gathered}
\boldsymbol{U}_{i b}(s, t)=\int_{V} \boldsymbol{u}(x, t) \delta_{h}(\boldsymbol{X}(s, t)-\boldsymbol{x}) d \boldsymbol{x}, \\
\boldsymbol{f}(\boldsymbol{x}, t)=-\int_{\Gamma} \boldsymbol{F}(s, t) \delta_{h}(\boldsymbol{X}(s, t)-\boldsymbol{x}) d s
\end{gathered}
$$




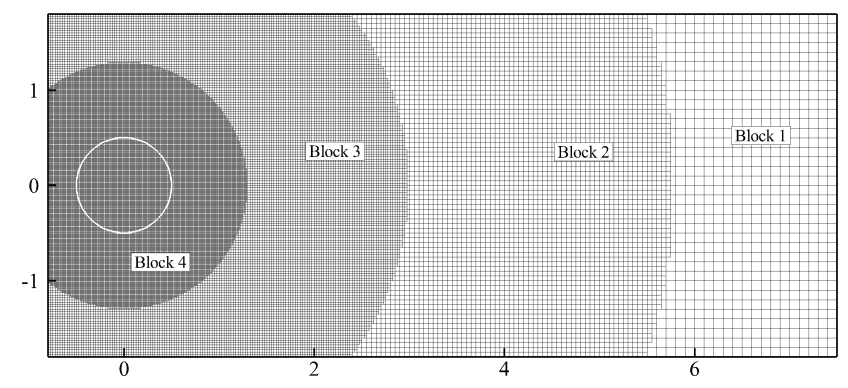

Figure 1. Geometry-adaptive mesh strategy.

where $\boldsymbol{u}$ is the fluid velocity, $\boldsymbol{X}$ is the coordinates of structural nodes, $\boldsymbol{x}$ is the coordinates of fluid, $s$ is the arc coordinate, and $V$ is the fluid domain, and $\Gamma$ is the structure domain. The smooth function $\delta_{h}$ is used to approximate the Dirac delta function. In this paper, the four-point delta function introduced by Peskin (Peskin 2002 ) is used.

The heat transferred from the immersed boundary to the fluid can be written as (Ren et al. 2012)

$$
q=\int_{\Gamma} Q(s, t) \delta_{h}(\boldsymbol{X}(s, t)-\boldsymbol{x}) d s,
$$

where $Q$ is the virtual boundary heat flux. When the temperature boundary is used, then $Q$ can be calculated using the penalty immersed-boundary method, expressed as

$$
Q=\alpha_{T}\left(T_{i b}-T_{0}\right),
$$

where $\alpha_{T}$ is a large factor, $T_{0}$ is the boundary condition on the immersed boundary, and $T_{i b}$ is the temperature of the virtual boundary, interpolated by

$$
T_{i b}(s, t)=\int_{V} T(x, t) \delta_{h}(\boldsymbol{X}(s, t)-\boldsymbol{x}) d \boldsymbol{x} .
$$

\section{VALidations}

\subsection{Non-Newtonian Power-Law Fluid Flow and Heat Transfer around a Stationary Cylinder}

Non-Newtonian power-law fluid flow and heat transfer from a stationary cylinder is considered to validate the accuracy of the present numerical method in handling FSI and heat transfer. The two-dimensional power-law fluid inlets with a uniform flow $U_{0}$ over a stationary circular cylinder (of diameter $D$ ), with the computational domain extending from $(-15 D,-5 D)$ to $(25 D, 5 D)$ and the cylinder located at the origin.

In order to improve the computational efficiency and validate the geometry-adaptive technique utilized in present in-house code, four blocks are used in the simulations, as shown in Figure 1, and the most refined mesh size of the fluid domain is $D / 80$. The non-dimensional parameters that control this problem include the Reynolds number (Re) and the Prandtl number (Pr), which are defined as $\operatorname{Re}=\rho_{0} D^{n} U_{0}^{2-n} / m, \quad \operatorname{Pr}=$ $c_{p} m\left(U_{0} / D\right)^{n-1} / \kappa$. The drag coefficient, lift coefficient, and Strouhal number are defined as $C_{D}=$ $-\sum F_{x} /\left(0.5 \rho U_{0}^{2} D\right), C_{L}=\sum F_{y} /\left(0.5 \rho U_{0}^{2} D\right), \mathrm{St}=D /\left(U_{0} T\right)$, where $T$ is the vortex shedding period, and $F_{x}$ and $F_{y}$ are the horizontal and vertical components of $\boldsymbol{F}$ acting on the cylinder calculated by Equation (11).

Firstly, we consider the unsteady flow of non-Newtonian power-law flow over a cylinder at $\operatorname{Re}=100$ (at which the flow patterns show von-Kármán periodic vortex shedding behind the cylinder) to validate the geometryadaptive fluid solver in terms of handling FSI problem. In order to achieve the early von-Kármán vortex shedding, a vertical velocity perturbation is introduced into the initial inlet boundary. Four different indexes $n=0.6,1.0$, and 1.4 are calculated. Table 1 shows the present results of St, $C_{D, m}$ (mean drag coefficient) and $C_{L}$ as well as results from the literature. It shows that the present results agree well with these other values.

After the validation of the fluid solver in computing FSI problems involving non-Newtonian flow, we further consider the steady flow and heat transfer around a cylinder. The average Nusselt number $(N u)$ used for quantitative comparison with the available data from the literature are defined as $N u=\frac{1}{2 \pi} \int_{0}^{2 \pi} N u(\theta) \mathrm{d} \theta$, where 
L. Wang and F.-B. Tian, A hybrid immersed boundary-lattice Boltzmann and finite difference method...

Table 1. A uniform flow over a stationary cylinder at $\mathrm{Re}=100$. St: Strouhal number; $C_{D, m}$ : drag coefficient; $C_{L}$ : lift coefficient.

\begin{tabular}{|c|c|c|c|c|}
\hline$n$ & Sources & St & $C_{D, m}$ & $C_{L}$ \\
\hline \multirow{3}{*}{0.6} & Present & 0.182 & 1.258 & 0.375 \\
\hline & Patnana et al. (Patnana et al. , 2009) & 0.180 & 1.180 & - \\
\hline & Tian et al. (Tian et al., 2014) & 0.188 & 1.179 & 0.367 \\
\hline \multirow{4}{*}{1.0} & Present & 0.164 & 1.415 & 0.349 \\
\hline & Patnana et al. (Patnana et al. , 2009) & 0.164 & 1.341 & 0.325 \\
\hline & Tian et al. (Tian et al. , 2014) & 0.160 & 1.430 & 0.360 \\
\hline & Wang et al. (Wang et al. 2017) & 0.161 & 1.450 & 0.310 \\
\hline \multirow{3}{*}{1.4} & Present & 0.159 & 1.546 & 0.345 \\
\hline & Patnana et al. (Patnana et al. , 2009) & 0.150 & 1.497 & - \\
\hline & Tian et al. (Tian et al. , 2014) & 0.161 & 1.523 & 0.356 \\
\hline
\end{tabular}

Table 2. Averaged Nusselt number $(N u)$ for forced convection heat transfer from a stationary cylinder to power-law fluids at $\operatorname{Pr}=1.0$.

\begin{tabular}{ccccc}
\hline Re & Sources & $\boldsymbol{n}=\mathbf{0 . 8}$ & $\boldsymbol{n}=\mathbf{1 . 0}$ & $\boldsymbol{n}=\mathbf{1 . 4}$ \\
\hline \multirow{4}{*}{10} & Present & 2.089 & 2.038 & 1.973 \\
& Bharti et al. (Bharti et al. 2008) & 2.123 & 2.060 & 1.973 \\
& Tian et al. (Tian et al., 2014) & 2.208 & 2.150 & 2.075 \\
& Soares et al. (Soares et al., 2005) & 2.116 & 2.058 & 1.973 \\
\hline \multirow{4}{*}{40} & Present & 3.714 & 3.588 & 3.401 \\
& Bharti et al. (Bharti et al. 2008) & 3.830 & 3.653 & 3.400 \\
& Tian et al. (Tian et al., 2014) & 3.923 & 3.769 & 3.554 \\
& Soares et al. (Soares et al., 2005) & 3.736 & 3.570 & 3.325 \\
\hline
\end{tabular}

$N u(\theta)$ is the local Nusselt number on the surface of the cylinder, it can be evaluated using the temperature field according to $N u(\theta)=-\partial T / \partial \boldsymbol{n}_{s}$, where $\boldsymbol{n}_{s}$ is the unit vector normal to the surface of the cylinder.

The average Nusselt number from the current computation along with the available data from the literature of two Reynolds number $(\operatorname{Re}=10$ and 40$)$ and three indexes $(n=0.8,1.0$, and 1.4) are presented in Table 2 . It is found that the present results are in good agreement with other published data. With the increase of $n$ from 0.8 to 1.4 , the average Nusselt number decreases slightly at both Reynolds numbers. Comparisons between the Reynolds numbers of 10 and 40 show that the average Nusselt number changes more remarkably with larger Reynolds numbers. It is because of the enhancement of the heat convection effects under the larger Reynolds number.

\subsection{Flow around a sphere at $\mathrm{Re}=\mathbf{1 0 0 0 0}$}

In this section, flow around a sphere at a Reynolds number of $10000\left(\operatorname{Re}=\rho_{f} U D / \nu\right.$, where $U$ is the inlet velocity and $D$ is the diameter of the sphere) is considered to validate the incorporation of LES with the IB-LBM solver. The mean drag over $100 U / D$ is 0.41 which is close to the previous numerical result 0.39 (Constantinescu and Squires, 2003) and experimental results 0.40 (Achenbach, 1972). Fig. 2 shows the power-spectraldensity of the turbulent kinematic energy measured at $(3.0 D, 0.6 D, 0)$. It shows that the $-5 / 3$ Kolmogorov law is well reproduced as reported in Ref. (Xu et al. 2018), which confirms the LES model used in the present solver.

\section{BUSHFIRE MODELLING}

Catastrophic bushfire that destroys the assets and multiple facilities happened many times all over the world in the last two decades. The majority of the most damaging fires involve wind and terrain, in order to predict 
L. Wang and F.-B. Tian, A hybrid immersed boundary-lattice Boltzmann and finite difference method...

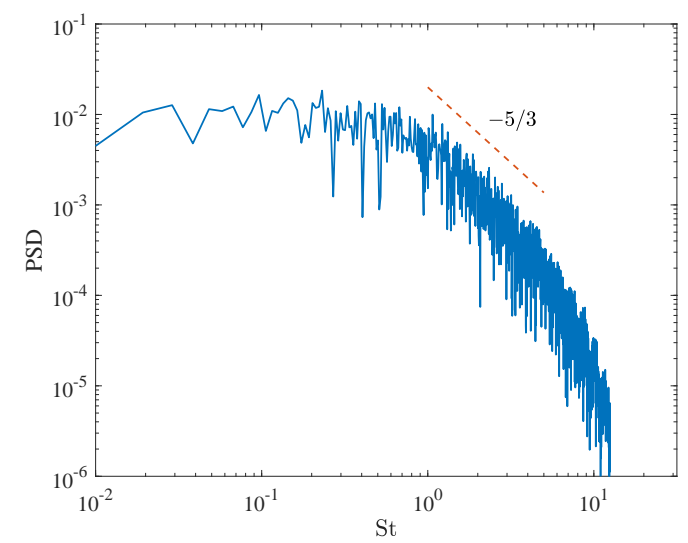

Figure 2. Power-spectral-density of the turbulent energy measured at $(3.0 D, 0.6 D, 0)$.
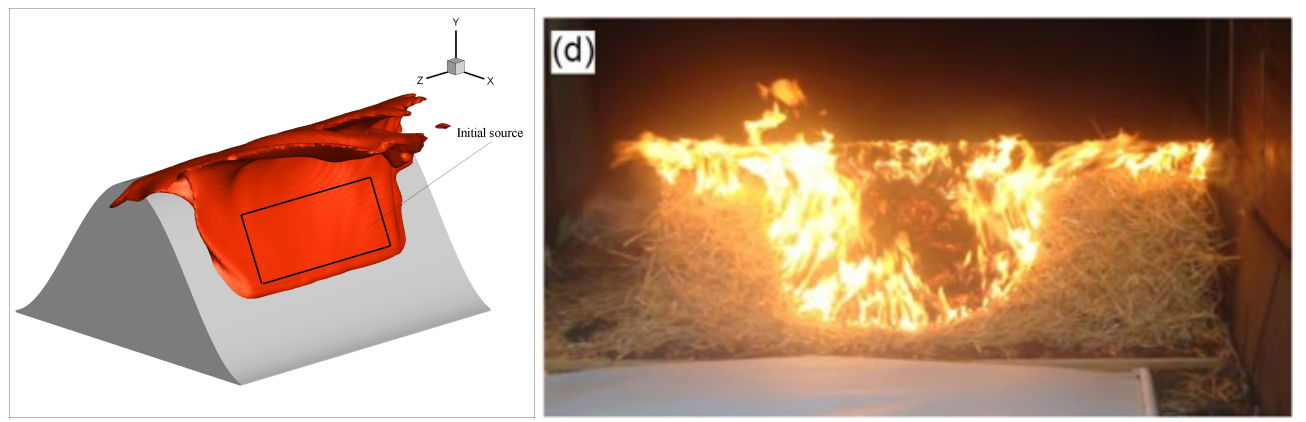

Figure 3. Qualitative comparison of the temperature filed: present numerical results (left) and experimental results from Ref. (Sharples and McRae, 2011).

the spread process of bushfire in various geographical and weather conditions, it is necessary to improve understanding of the physical mechanisms of bushfire-terrain-wind interaction. Here, bushfire spread across a lee-slope in a uniform flow is considered. The simulation is conducted at a Reynolds number of 10000 and Richard number of 0.5. A qualitative comparison of the current result and that from Ref. (Sharples and McRae, 2011) is presented in Fig. 3. The results show that that the slope plays an important role in the bushfire spread. Specifically, the heat spreads to the two sides across the top of the slope, which will certainly induce a fast bushfire spread along the ridge of the slope. This phenomenon is well captured by the present numerical method, which agrees with the observation in the experiment.

\section{CONCLUSIONS}

A hybrid immersed boundary-lattice Boltzmann and finite difference method is proposed for the bushfire simulation. The integration of spatial adaptivity, thermal finite difference method, and fluid flow immersed boundary-lattice Boltzmann method is validated by flow around a circular cylinder in power-law fluid, heat convection around a circular cylinder in a uniform flow and flow around a sphere at $\mathrm{Re}=10000$. A demonstration considering bushfire-wind-terrain interaction is also presented. The further study on bushfire-windterrain interaction will be presented in the future.

By using an immersed boundary method, the present numerical method has a superiority in handling complex and moving boundaries, which makes it potentially applicable for many other related engineering problems, such as building ventilation and battery cooling. Incorporating wall model into the present method is a future work to make it more efficient for high Reynolds number problems. 
L. Wang and F.-B. Tian, A hybrid immersed boundary-lattice Boltzmann and finite difference method...

\section{REFERENCES}

Abel, S., K. Prasad, and A. Mahaboob (2005). Buoyancy force and thermal radiation effects in MHD boundary layer viscoelastic fluid flow over continuously moving stretching surface. International Journal of Thermal Sciences 44(5), 465476.

Achenbach, E. (1972). Experiments on the flow past spheres at very high Reynolds numbers. Journal of Fluid Mechanics 54(3), 565-575.

Alexander, F. J., S. Chen, and J. Sterling (1993). Lattice Boltzmann thermohydrodynamics. Physical Review E 47(4), R2249.

Bharti, R., P. Sivakumar, and R. Chhabra (2008). Forced convection heat transfer from an elliptical cylinder to powerlaw fluids. International Journal of Heat and Mass Transfer 51(7), 1838-1853.

Chen, S. and G. D. Doolen (1998). Lattice Boltzmann method for fluid flows. Annual review of fluid mechanics 30(1), 329-364.

Chen, Y., H. Ohashi, and M. Akiyama (1997). Two-parameter thermal lattice BGK model with a controllable prandtl number. Journal of scientific computing 12(2), 169-185.

Constantinescu, G. S. and K. D. Squires (2003). Les and des investigations of turbulent flow over a sphere at $\mathrm{Re}=$ 10,000. Flow, Turbulence and Combustion 70(1-4), 267-298.

Guo, Z. and C. Shu (2013). Lattice Boltzmann method and its applications in engineering. World Scientific.

Guo, Z. and C. Zheng (2008). Analysis of lattice Boltzmann equation for microscale gas flows: relaxation times, boundary conditions and the knudsen layer. International Journal of Computational Fluid Dynamics 22(7), 465-473.

Inamuro, T., M. Yoshino, H. Inoue, R. Mizuno, and F. Ogino (2002). A lattice Boltzmann method for a binary miscible fluid mixture and its application to a heat-transfer problem. Journal of Computational Physics 179(1), 201-215.

Kim, Y. and C. S. Peskin (2007). Penalty immersed boundary method for an elastic boundary with mass. Physics of Fluids 19(5), 053103.

Lilly, D. K. (1992). A proposed modification of the Germano subgrid-scale closure method. Physics ofFluids A: Fluid Dynamics 4(3), 633-635.

Mittal, R., H. Dong, M. Bozkurttas, F. Najjar, A. Vargas, and A. Von Loebbecke (2008). A versatile sharp interface immersed boundary method for incompressible flows with complex boundaries. Journal of Computational Physics 227(10), 4825-4852.

Patnana, V. K., R. P. Bharti, and R. P. Chhabra (2009). Two-dimensional unsteady flow of power-law fluids over a cylinder. Chemical Engineering Science 64(12), 2978-2999.

Peng, Y., C. Shu, and Y. Chew (2003). Simplified thermal lattice Boltzmann model for incompressible thermal flows. Physical Review E 68(2), 026701.

Peskin, C. S. (2002). The immersed boundary method. Acta Numerica 11, 479-517.

Qian, Y. (1993). Simulating thermohydrodynamics with lattice BGK models. Journal of Scientific Computing 8(3), 231242.

Ren, W., C. Shu, J. Wu, and W. Yang (2012). Boundary condition-enforced immersed boundary method for thermal flow problems with dirichlet temperature condition and its applications. Computers \& Fluids 57, 40-51.

Sharples, J. J. and R. H. McRae (2011). Atypical bushfire spread driven by the interaction of terrain and extreme fire weather. In Proceedings of Bushfire CRC \& AFAC 2011 Conference Science Day, Volume 1.

Soares, A., J. Ferreira, and R. Chhabra (2005). Flow and forced convection heat transfer in crossflow of non-newtonian fluids over a circular cylinder. Industrial \& Engineering Chemistry Research 44(15), 5815-5827.

Tian, F.-B., R. P. Bharti, and Y.-Q. Xu (2014). Deforming-spatial-domain/stabilized space-time (DSD/SST) method in computation of non-newtonian fluid flow and heat transfer with moving boundaries. Computational Mechanics 53(2), 257-271.

Tian, F.-B., H. Dai, H. Luo, J. F. Doyle, and B. Rousseau (2014). Fluid-structure interaction involving large deformations: 3D simulations and applications to biological systems. Journal of Computational Physics 258, 451-469.

Tian, F.-B., H. Luo, L. Zhu, J. C. Liao, and X.-Y. Lu (2011). An efficient immersed boundary-lattice Boltzmann method for the hydrodynamic interaction of elastic filaments. Journal of computational physics 230(19), 7266-7283.

Wang, L., G. M. Currao, F. Han, A. J. Neely, J. Young, and F.-B. Tian (2017). An immersed boundary method for fluidstructure interaction with compressible multiphase flows. Journal of Computational Physics 341, 131-151.

Wang, L. and F.-B. Tian (2018). Heat transfer in non-newtonian flows by a hybrid immersed boundary-lattice boltzmann and finite difference method. Applied Sciences 8(4), 559.

Xu, L., F.-B. Tian, J. Young, and J. C. Lai (2018). A novel geometry-adaptive cartesian grid based immersed boundarylattice Boltzmann method for fluid-structure interactions at moderate and high Reynolds numbers. Journal of Computational Physics 375, 22-56.

$\mathrm{Yu}, \mathrm{D}$., R. Mei, and W. Shyy (2002). A multi-block lattice Boltzmann method for viscous fluid flows. International journal for numerical methods in fluids 39(2), 99-120. 